\title{
Nonlinear performance characterization in an eight-pole quasi-elliptic bandpass filter
}

\author{
J Mateu ${ }^{1}$, C Collado ${ }^{2}$, O Menéndez ${ }^{2}$ and J M O'Callaghan ${ }^{2}$ \\ ${ }^{1}$ Centre Tecnològic de Telecomunicacions de Catalunya, Edifici Nexus, Gran Capità, \\ 2nd Floor, Room 202-203, 08034 Barcelona, Spain \\ ${ }^{2}$ Universitat Politècnica de Catalunya, Department of Signal Theory and Communications, \\ Campus Nord UPC, D3-Jordi Girona, 1-3, 08034 Barcelona, Spain \\ E-mail: jordi.mateu-mateu@cttc.es and joano@tsc.upc.es
}

Received 17 December 2003

Published 14 April 2004

Online at stacks.iop.org/SUST/17/S359

DOI: $10.1088 / 0953-2048 / 17 / 5 / 054$

\begin{abstract}
In this work we predict the nonlinear behaviour of an eight-pole quasi-elliptic bandpass high temperature superconducting (HTS) filter with an equivalent circuit extracted from intermodulation measurements performed at the centre of the filter passband. We present measurements that show that the equivalent circuit is able to predict the intermodulation products produced by the filter when driven by two in-band or out-of-band sinusoidal signals. Numerical techniques based on harmonic balance are used to extract the elements of the equivalent circuit and to simulate its nonlinear performance.
\end{abstract}

\section{Introduction}

Microwave nonlinearities in superconductor materials are still an important limitation which exclude the use of these materials in many applications at microwave frequencies. These nonlinearities are enhanced when there are high current densities in the HTS, and are therefore more prominent in planar resonators and filters where magnetic field wraps around a thin film of superconducting material. These high current densities in the HTS lead to generation of harmonics and intermodulation distortion (IMD) which may have a negative impact on the performance of microwave superconductive devices. Among these devices are the HTS preselect bandpass filters used in base stations of cellular communication systems and other civil and military applications. These filters are placed at the front of a receiver to block interfering signals outside the receiver band. They should therefore be able to simultaneously handle weak in-band and strong out-of-band signals. This ability might be limited by the IMD of the filter, which might generate spurious signals that may degrade the receiver's performance. Quantifying this degradation is crucial in determining if the use of the HTS filter in the receiver is beneficial. One possible way of doing this is by having a nonlinear equivalent circuit of the HTS filter on which one could predict the effects of the interfering signals.
In this paper we describe an approach for extracting a nonlinear equivalent circuit of the HTS filter and simulating its performance. We have tested the approach on a planar, eightpole quasi-elliptic (QE) filter. We show that the agreement between simulations and measurements of IMD is not limited to the frequencies within the passband, but extends to the transition bands at either side of the passband. The maximum frequency separation from the passband edge at which agreement can be checked is limited by our measurement (dynamic range) constraints.

Finally, we also discuss how to relate the values of the elements of the equivalent circuit with the nonlinear properties of the HTS film derived from measurements of unpatterned films in rutile-loaded resonators.

\section{Filter development and fabrication}

For the filter design we have considered an eight-pole QE filter [1], as shown in figure 1. The frequency response of the filter has transmission zeros at both edges of the passband, which are realized because the cross-coupling between the resonators 3 and 6 has a different sign to that of the sequential couplings (that is the couplings between the resonators $i$ and $i+1)$. 
Table 1. Coupling coefficients and external quality factor resulting from the synthesis process.

\begin{tabular}{llllll}
\hline$M_{12}=M_{78}$ & $M_{23}=M_{67}$ & $M_{34}=M_{56}$ & $M_{45}$ & $M_{36}$ & $Q_{\mathrm{ei}}=Q_{\mathrm{eo}}$ \\
\hline 0.00560 & 0.00426 & 0.00367 & 0.00559 & -0.00167 & 169.085 \\
\hline
\end{tabular}

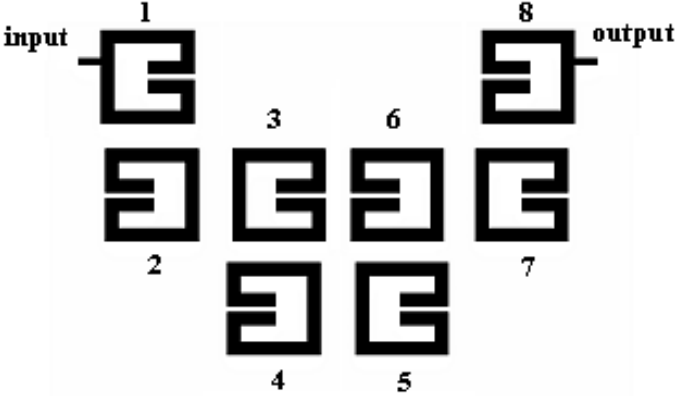

Figure 1. Outline of an eight-pole microstrip bandpass filter with a single pair of transmission zeros.

The resonant frequency of the basic resonator has been set to about $1.9725 \mathrm{GHz}$ by means of electromagnetic simulations $^{3}$. Another important parameter which defines the performance of the filter is the unloaded quality factor $\left(Q_{0}\right)$ of the resonators which may also be obtained from electromagnetic simulation taking into account the material properties. The unloaded quality factor results $\sim 50000$. The bandpass of the filter is $15 \mathrm{MHz}$ and the transmission zeros are 17.25 MHz apart and symmetrically placed about the central frequency [2]. The filter has been designed to have $-15 \mathrm{~dB}$ passband return losses. From the specification of the filter and using the synthesis procedure detailed in [2], we obtain the coupling coefficients between resonators $\left(M_{i j}\right)$ and the external quality factors of the input and output ports $\left(Q_{\mathrm{ei}}\right.$, $\left.Q_{\mathrm{eo}}\right)$, which are listed in table 1 . The solid grey curve in figure 2 shows the synthesized response. To produce a layout that implements these parameters, we follow the procedure described in [3]. The dashed curve in figure 2 shows the response of the filter design obtained by using electromagnetic simulations (see footnote 3 ).

The superconducting filter was fabricated using a 2 inch double-sided $700 \mathrm{~nm} \mathrm{YBa} \mathrm{Cu}_{3} \mathrm{O}_{7-\delta}$ (YBCO) thin film deposited onto a $508 \mu \mathrm{m}$ thick $\mathrm{MgO}$ substrate. Both sides of the YBCO are metallized with $200 \mathrm{~nm}$ of gold deposited in situ on the side to be patterned and ex situ on the ground plane side. The film was patterned using standard photolithography and wet chemical etching, and was tuned using sapphire rods. Figure 2 shows the frequency response of the filter after the tuning process (solid curve).

\section{Equivalent circuit}

In our equivalent circuit, we model the open-loop resonators as straight half-wave lines (figure 3). The equivalent microstrip lines are made to replicate the current density in the central segment of the open loop resonator where current density is maximum. This should, in turn, make the nonlinear effects in the transmission line similar to those in the open-loop resonator, since these effects are mainly produced where the current density is largest.

\footnotetext{
3 Momentum Agilent Technologies.
}

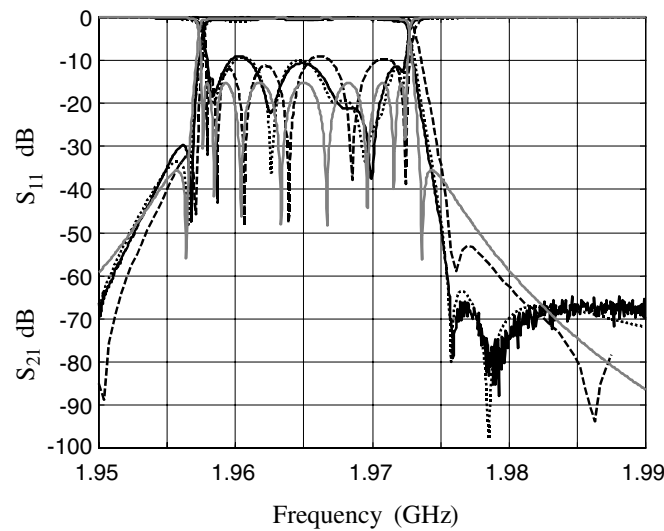

Figure 2. Solid grey curve: synthesis response. Dashed curve: simulated filter response using a full wave electromagnetic simulator. These responses have been shifted by $7.5 \mathrm{MHz}$ to make them match with the measured one. Solid curve: measured filter response after the tuning process. Dotted curve: modelled filter response using the equivalent circuit.

a)
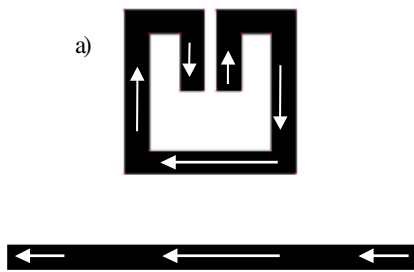

b)

Figure 3. (a) Basic resonator. The white arrows represent the current amplitude at resonance. Note that in the middle of the resonator the current is largest. (b) Equivalent half-wave straight transmission line.

To analyse the spatially distributed nonlinearities in a HTS filter, we discretized all the equivalent half-wave resonators of the filter by cascading many identical two-ports, each one modelling a segment of the transmission line much smaller than a wavelength [4]. Figure 4 shows the equivalent circuit of an elemental segment of the transmission line, where the distributed parameters $L_{0}, R_{0}, C$ and $G$ depend on the geometric cross section of the line and on the material properties [5]. The nonlinear effects of the HTS in these lines are modelled by a dependence of the resistance and inductance per unit of length on the total current through the line $(i)$ : $R(i)=R_{0}+\Delta R(i), L(i)=L_{0}+\Delta L(i)[6]$, where $\Delta R(i)$ and $\Delta L(i)$ can be related to the variation of the penetration depth with the volume current density, $\lambda_{L}(j)[5,6]$.

The nonlinear equivalent circuit of a single resonator will be made up of many elemental segments like the one shown in figure 4. To form the nonlinear equivalent circuit of the whole filter we use eight identical sub-circuits-one for each resonator-and couple them using impedance inverters [7]. Note that this implies that the equivalent circuit elements of the resonators in the filter do not change significantly with respect to the values that they have when the resonator is isolated. 
Nonlinear performance characterization in an eight-pole quasi-elliptic bandpass filter

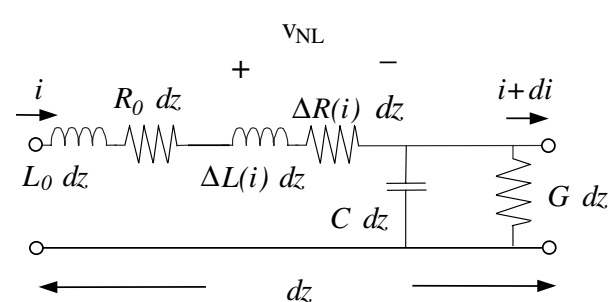

Figure 4. Equivalent circuit valid for a small segment of the HTS nonlinear transmission line of length $\mathrm{d} z$.

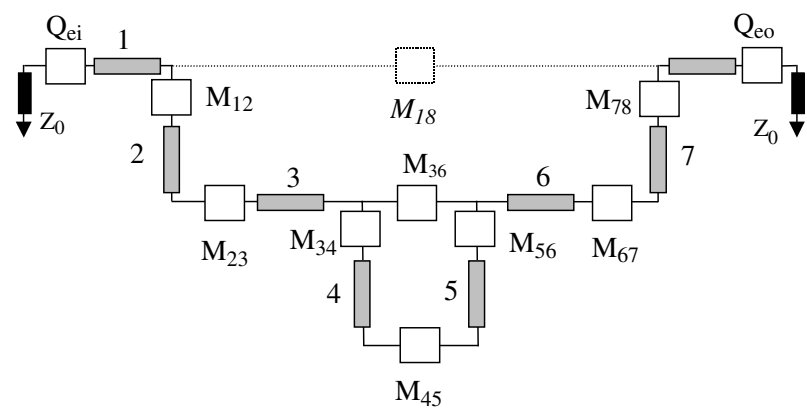

Figure 5. Outline of the equivalent bandpass filter circuit model. The square boxes represent the coupling between resonators $\left(M_{i j}\right.$, $Q_{\text {ei }}$ and $Q_{\text {eo }}$ ), the rectangular grey boxes represent the halfwave transmission line resonators. We also take into account parasitic couplings like $M_{18}$ (dashed box), which are required to fit the filter response (figure 2, solid curve). Of these couplings, only $M_{18}$ is shown in this figure, to preserve its clarity, but the couplings $M_{16}$, $M_{24}, M_{35}, M_{46}, M_{57}, M_{38}$ and $M_{68}$ are also considered.

This happens because the couplings among resonators are very weak. Otherwise, both the linear and nonlinear circuit elements in figure 4 would be affected.

The resulting equivalent circuit is shown in figure 5 . Note that it includes impedance inverters to model the couplings taken into account in the filter design (that is, $M_{36}$ and $M_{i, i+1}$ ) as well as other parasitic coupling effects. The latter are unavoidable in microstrip technology, where the resonators are open structures and any set of two resonators in the same layout may exhibit coupling effects [1]. Some of these parasitic couplings have to be taken into account for an accurate fit of the equivalent circuit with the frequency response of the filter.

\section{Experimental fit of the equivalent circuit elements}

A critical first step in the determination of the equivalent circuit is to find the values of its linear circuit elements, including the coupling between resonators. The fit of the linear elements has a strong effect on the accuracy of the nonlinear ones, since it determines the amplitudes of the currents in the resonators. To find these values, we assume that the circuit is fully linear $(\Delta R(i)$ and $\Delta L(i)$ are zero) and adjust the linear circuit elements to fit the frequency dependence of the measured small-signal $S$ parameters of the filter after the tuning process. To do that, we have used a gradient optimization routine that adjusts the couplings and resonant frequencies to fit the frequency response. The routine fits couplings in table 1, some parasitic couplings $\left(M_{13}, M_{16}, M_{18}, M_{24}, M_{35}, M_{46}, M_{57}\right.$, $M_{38}$ and $\left.M_{68}\right)$ and the resonant frequency of all the resonators. The initial values for this routine are the couplings in table 1, a resonant frequency of $1.965 \mathrm{GHz}$ for all resonators, and all

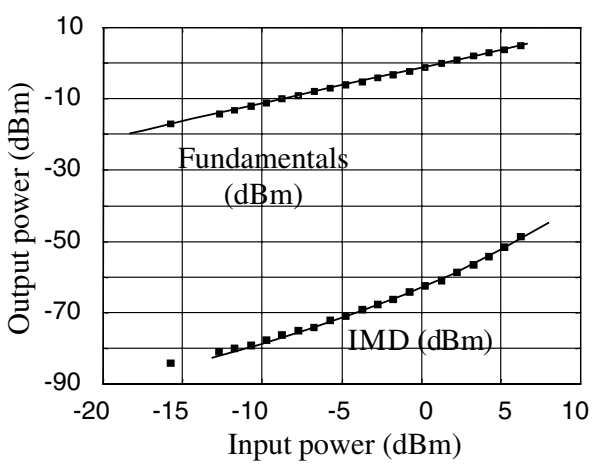

Figure 6. Results of measurements and simulations. Square marks indicate the measured power of the intermodulation products $2 f_{2}-f$ and the power at the output of the filter at fundamental frequencies as a function of the source power when the power of both sources was increased simultaneously. The lines in the figure are the results obtained with harmonic balance and the equivalent circuit of figure 5 .

parasitic couplings are assumed to be zero. After the gradient optimization, we find that some of the designed couplings (table 1) change up to $30 \%$, the resonant frequencies up to $0.2 \%$ and the parasitic couplings are about 100 times lower than the ones in table 1. Figure 2 shows the result of the adjustment: the solid curve (measured $S_{21}$ and $S_{11}$ ) closely fits the values predicted by the linear circuit (dotted curve).

Once the linear elements are determined, we can simulate the effects of the nonlinear ones $(\Delta R(i)$ and $\Delta L(i))$ using harmonic balance $(\mathrm{HB})[2,4]$. Even though the equivalent circuit has many elements, the simulation is fast enough to do an iterative fit of $\Delta R(i), \Delta L(i)$ to match the simulation results to the measured intermodulation data. The measurements to obtain these data have been performed by placing the two fundamental tones $\left(f_{1}, f_{2}\right)$ symmetrically at $50 \mathrm{kHz}$ above and below the central frequency of the passband of the filter, and they are kept balanced in the whole experiment. Figure 6 shows the results and the fit performed with harmonic balance.

The parameters $\Delta R(i), \Delta L(i)$ can be fitted by either assuming that the nonlinear effects are due to the resistive part $(\Delta R(i))$ or to the reactive part $(\Delta L(i))$. However in this experiment we assume that resistive nonlinearities are negligible $[6,8]$, and then we extract $\Delta L(i)$. Doing that, the resulting nonlinear parameter $\Delta L(i)$ is $7.93 \times 10^{-12}|i|^{0.2}+$ $22.5 \times 10^{-12}|i|^{2} \mathrm{H} \mathrm{m}^{-1}$. The solid lines of figure 6 represent the results of applying $\Delta L(i)$ obtained. Note that at low input power the slope of the intermodulation products in figure 6 is dominated by the first term of the nonlinear inductance per unit of length $\left(7.93 \times 10^{-12}|i|^{0.2} \mathrm{H} \mathrm{m}^{-1}\right)$, whereas at higher input power the slope increases because of the contribution of the second term of the nonlinear inductance per unit of length $\left(22.5 \times 10^{-12}|i|^{2} \mathrm{H} \mathrm{m}^{-1}\right)$.

\section{Experimental cross-check}

Once the nonlinearities of the filter have been characterized from the measurements in the centre of the band, we have extended our experiments by measuring the intermodulation products in the bandpass and transition band of the filter. In this experiment the frequency separation between the two fundamental tones is kept at $100 \mathrm{kHz}$ and their power to $5 \mathrm{dBm}$. Figure 7 shows the results. 


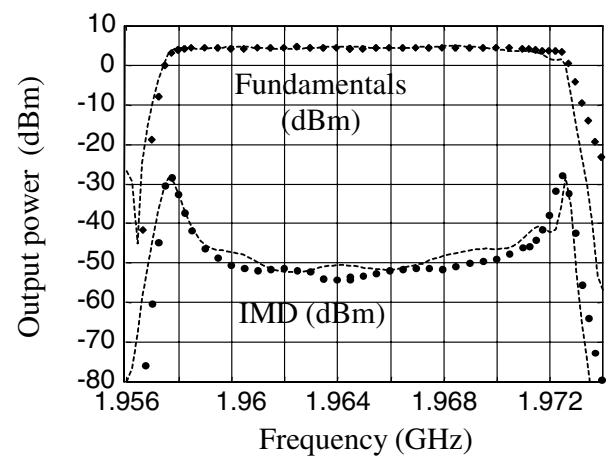

Figure 7. Results of measurements and simulations. Symbols measured power of intermodulation products at $2 f_{2}-f_{1}$ and fundamental tones $\left(f_{1}\right.$ and $\left.f_{2}\right)$ as a function of the source frequency when the power of both sources is $5 \mathrm{dBm}$. The separation between the frequency components is kept at $100 \mathrm{kHz}$ in the whole experiment. The lines in the figure are the results of simulations performed with parameters obtained with harmonic balance and applying the equivalent circuits of figures 4 and 5 .

The symbols represent the measured values, whereas the dashed curves represent the predicted results obtained from the equivalent circuit that fit the measurements of figure 6 . We see that the intermodulation measurements in figure 7 are predicted correctly. In other words, the equivalent circuit and the nonlinear model with its nonlinear extracted parameters allows us to predict nonlinear behaviour in the whole band of the filter. Note also that this is true even at the edges of the passband where the circulating power is higher and in the transition band where the power of the fundamental tones might be very unbalanced. This result is relevant since, in a receiver, the interfering signals may be close to the edges of the passband.

\section{Discussion}

We have recently studied the nonlinearities of unpatterned HTS thin films [9] from the vendor that supplied the sample used to fabricate the filter. From those experiments we found that intermodulation properties can change significantly from sample to sample. This has also been observed in many published works $[10,11]$. From the results of one of the three samples measured in [9] $\left(\mathrm{HTS}_{1}\right)$, we predict the dependence of the inductance and resistance per unit length $(\Delta R(i)$ and $\Delta L(i))$ in a cross section of a microstrip line like the ones in the resonators of the filter. That is $1 \mathrm{~mm}$ width and $700 \mathrm{~nm}$ thick microstrip over a $508 \mu \mathrm{m}$ thick $\mathrm{MgO}$ dielectric. This results in $\Delta R(i)=2.65 \times 10^{-3}|i|^{0.2} \Omega \mathrm{m}^{-1}$ and $\Delta L(i)=3.8 \times 10^{-12}|i|^{0.2} \mathrm{H} \mathrm{m}^{-1}$. Note that, according to these values, the reactive term is dominant over the resistive one $(\Delta R \ll \omega \Delta L)[6]$. Comparing the inductance per unit of length extracted from the experiment above with the one obtained from unpatterned measurements in [9], we see that at low currents both results are within a factor of two.

On the other hand, as mentioned in section 3, the nonlinear parameters of the equivalent circuit $(\Delta R(i)$ and $\Delta L(i))$ can be related to the variation of the penetration depth on the volume current density. In [6] the nonlinear effects are explained by assuming a variation of the penetration depth as $\lambda_{L}(T, j)=$ $\lambda_{L}(T, j)[1+0.5 f(T, j)]$, with $f(T, j)=\left(j / j_{\mathrm{IMD}}\right)^{2}$. For $\operatorname{HTS}_{1}[9]$, we proposed $f(T, j)=\left|j / j_{\mathrm{IMD} 1}\right|^{0.2}$, where $j_{\mathrm{IMD} 1}=$
$7.3 \times 10^{18} \mathrm{~A} \mathrm{~m}^{-2}$ [2]. In the experiments of [9] the maximum volume current density reached at the superconductor was $\sim 5 \times 10^{8} \mathrm{~A} \mathrm{~m}^{-2}$

For the sample used in this work we propose $f(T, j)=$ $\left|j / j_{\mathrm{IMD} 1}\right|^{0.2}+\left|j / j_{\mathrm{IMD} 2}\right|^{2}$, being $j_{\mathrm{IMD} 1}=2.65 \times 10^{17} \mathrm{~A} \mathrm{~m}^{-2}$ and $j_{\text {IMD2 }}=8 \times 10^{10} \mathrm{~A} \mathrm{~m}^{-2}$. In this case the volume current density in the strips of the filter at $-10 \mathrm{dBm}$ is nearly the same as the one in the cavity in [9], $\sim 5 \times 10^{8} \mathrm{~A} \mathrm{~m}^{-2}$. At these levels of volume current density the condition $\left|j / j_{\mathrm{IMD} 1}\right|^{0.2} \gg$ $\left|j / j_{\mathrm{IMD} 2}\right|^{2}$ holds, therefore $f(T, j) \approx\left|j / j_{\mathrm{IMD} 1}\right|^{0.2}$, which is consistent with the nonlinearities of $\mathrm{HTS}_{1}$ [9]. Although both samples have similar nonlinear behaviour (see their $\Delta L(i)$ ) their phenomenological parameter $j_{\text {IMD1 }}$ is quite different in both cases. This may be due to the low power dependence of the nonlinearities at such current density levels. On the other hand, at higher power, $5 \mathrm{dBm}$, the nonlinearities are dominated by a contribution of the second term, i.e. $\left|j / j_{\mathrm{IMD} 1}\right|^{0.2} \ll$ $\left|j / j_{\mathrm{IMD} 2}\right|^{2}$, as can be seen in figure 6 .

\section{Conclusion}

We have experimentally verified that the circuit model of the HTS bandpass filter and the numerical techniques based on HB are valid to both characterize and predict the nonlinear behaviour of the filter at the passband and transition band. Thus, this circuit model can be used to predict the nonlinear performance of real systems subject to broadband signals whose spectrums might partially leak to adjacent channels. This might happen in wireless base station receivers, one of the most important applications of HTS filters, where interference signals can appear in or out of the band of interest. In this work we have also seen that the nonlinear characterization is consistent with a previous characterization made on unpatterned thin films supplied by the same vendor.

\section{Acknowledgments}

This work was supported by the Spanish Ministry of Science and Technology (MCYT) under Project MAT2002-04551 and by the Generalitat de Catalunya (DURSI) under Grant 2001 SGR 0026

\section{References}

[1] Hong J-S and Lancaster M J 2002 Microstrip Filter for RF/Microwave Applications (Wiley Series in Microwave and Optical Engineering) (New York: Wiley)

[2] Mateu J 2003 Nonlinear distortion in microwave devices and systems UPC-PhD Thesis

[3] Hong J-S, Lancaster M J, Jedamzik D and Greed R B 1999 IEEE Trans. Microw. Theory Tech. 47 1656-63

[4] Collado C, Mateu J and O'Callaghan J M 2001 J. Supercond. $1457-64$

[5] Sheen D M and Ali S M 1991 IEEE Trans. Appl. Supercond. 1 $108-17$

[6] Dahm T and Scalapino D J 1997 J. Appl. Phys. 81 2002-9

[7] Matthaei G, Young L and Jones E 1964 Microwave Filters, Impedance-Matching Networks and Coupling Structures (New York: McGraw-Hill)

[8] Willemsen B A, Dahm T and Scalapino D J 1997 Appl. Phys. Lett. 71 3898-900

[9] Mateu J, Collado C, Menéndez O and O’Callaghan J M 2003 J. Supercond. $16873-80$

[10] Yoshitake T, Tahara S and Suzuki S 1995 Appl. Phys. Lett. 67 3963-5

[11] Hao L, Gallop J, Purnell A, Cohen L and Thiess S 2001 IEEE Trans. Appl. Supercond. $113411-4$ 